\title{
Infection congénitale probable au SRAS-CoV-2 chez le nouveau-né d'une femme atteinte de COVID-19 active
}

\author{
Maksim Kirtsman MDCM, Yenge Diambomba MD, Susan M. Poutanen MD MPH, Ann K. Malinowski MD, \\ Evangelia Vlachodimitropoulou MD PhD, W. Tony Parks MD, Laura Erdman MD PhD, Shaun K. Morris MD MPH, \\ Prakesh S. Shah MD MSc
}

- Citation : CMAJ 2020 June 15;192:E647-50. doi : 10.1503/cmaj.200821-f; diffusion hâtive le 14 mai 2020

Voir la version anglaise de l'article ici : www.cmaj.ca/lookup/doi/10.1503/cmaj.200821

$\mathbf{U}$ ne femme de 40 ans (gravida 2, para 1) a été admise dans un hôpital de soins tertiaires de Toronto, en Ontario. On a noté chez elle : neutropénie familiale, diabète de grossesse et problème d'infections bactériennes à répétition, dont 3 épisodes (sinusite, dermatite et bronchite) durant la présente grossesse qui sont rentrés dans l'ordre avec une antibiothérapie. Les détails quant à l'évolution de l'état de la mère et l'issue de la grossesse ont été publiés séparément en raison de son hémopathie ${ }^{1}$.

La patiente consultait pour myalgie, inappétence, fatigue, toux sèche et fièvre à $39^{\circ} \mathrm{C}$ au cours des 24 heures précédentes. Un test d'amplification en chaîne par polymérase couplée à une transcription inverse (RT-PCR, reverse transcription polymerase chain reaction) (dosage Allplex 2019-nCoV, Seegene) sur un prélèvement nasopharyngé s'est révélé positif à l'égard du coronavirus du syndrome respiratoire aigu sévère 2 (SRAS-CoV-2) que l'on soupçonnait. On n'a noté aucun signe d'anomalie fœtale durant la grossesse ou après l'admission. La femme n'a pas eu besoin d'assistance respiratoire au moment de l'accouchement. Une césarienne semi-urgente a été effectuée sous anesthésie régionale, avec mesures de précaution contre la transmission par aérosols, gouttelettes et contact, en raison d'une aggravation de la coagulopathie (élévation des taux de D-dimères et de fibrinogène et allongement du temps de thromboplastine partielle activée) et d'une baisse de la numération plaquettaire à 35 semaines et 5 jours de gestation. Une amniotomie a été pratiquée au moment de l'intervention. Le liquide amniotique était clair. Le nouveau-né de sexe masculin était vigoureux et n'a pas eu besoin de réanimation. Ses scores Apgar étaient de 9 à 1 minute de vie et de 9 à 5 minutes de vie, et son poids était de 2,93 kg.

Conformément au protocole de notre établissement, nous n'avons pas différé le clampage du cordon, et le nouveau-né a été immédiatement retiré du champ opératoire de façon stérile, vers un incubateur, à 2 mètres de distance dans la même pièce. Des prélèvements placentaires (maternel et fotal) ont été effectués. Le tissu placentaire a été envoyé au laboratoire pour PCR et examen histo-

\section{POINTS CLÉS}

- Les nouveau-nés de femmes porteuses d'une infection au coronavirus du syndrome respiratoire aigu sévère 2 (SRASCoV-2) confirmée ou présumée devraient subir des analyses de prélèvements nasopharyngés, placentaires et de sang de cordon le plus rapidement possible après la naissance, après une toilette minutieuse.

- Il faut consigner au dossier le moment et la technique des prélèvements et les types d'échantillons pour aider à distinguer l'acquisition d'une infection au SRAS-CoV-2 congénitale, périnatale ou postnatale chez les nouveau-nés.

pathologique. Des prélèvements nasopharyngés ont été effectués chez le nouveau-né le jour de la naissance, puis aux jours 2 et 7 , après une toilette minutieuse et avant tout contact avec la mère.

Les 3 prélèvements nasopharyngés du nouveau-né se sont révélés positifs à l'égard du SRAS-CoV-2 par RT-PCR; les analyses sérologiques quant à elles ont été positives au jour 4, et les analyses de selles au jour 7. Les résultats des analyses chez la mère et le nouveau-né, y compris celles des tests PCR pour le SRAS-CoV-2 par dosage Allplex 2019-nCoV, sont présentés au tableau supplémentaire S1 de l'annexe 1 (accessible en anglais ici : www.cmaj.ca/ lookup/suppl/doi:10.1503/cmaj.200821/-/DC1). En tant que mesures indirectes de la charge virale, les résultats détaillés, incluant les cycles seuils des tests $P C R$ pour les gènes cibles spécifiques (enveloppe, ARN polymérase ARN-dépendante et nucléocapside), sont présentés dans le tableau supplémentaire $\$ 2$ de l'annexe 1.

Chacune des 5 sections placentaires aléatoires a montré de multiples zones d'infiltration par les cellules inflammatoires et un infarctus précoce étendu. L'infiltrat inflammatoire se composait presque entièrement de macrophages CD68-positifs. Seuls des lymphocytes $T$ (CD3), des lymphocytes B (CD20) et des neutrophiles (CD15) dispersés ont été identifiés à l'examen immunohistochimique. L'infiltrat inflammatoire était pour une bonne part confiné à l'espace intervillaire, ce qui concorde avec une 


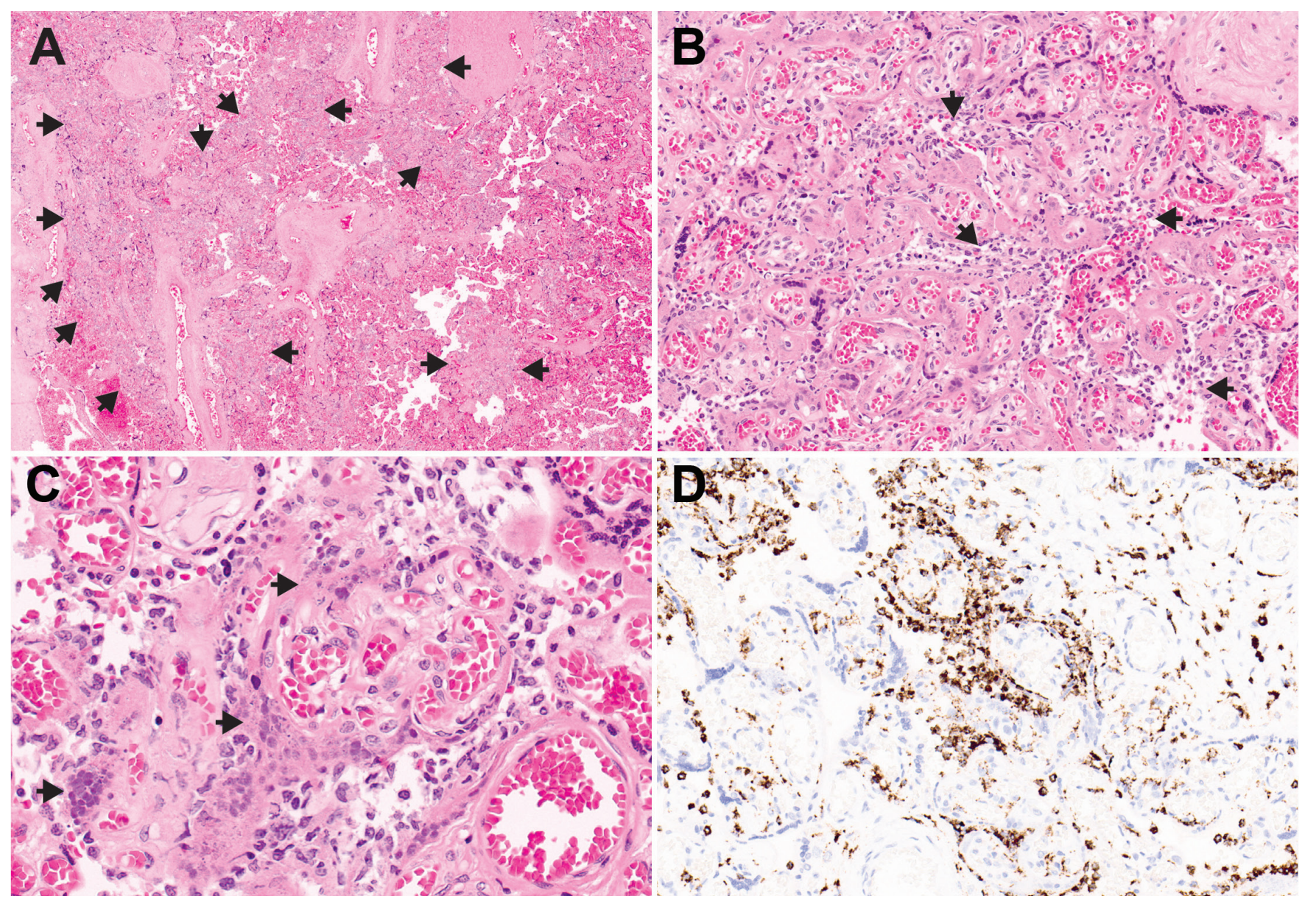

Figure 1 : Photomicrographies placentaires. (A) À l'imagerie de faible puissance, plusieurs zones vastes d'infiltrats cellulaires sont dispersées aléatoirement sur toute la surface (flèches). La zone la plus normale se trouve du côté droit de la diapo (hématoxyline-éosine, amplification originale $\times 2$ ). (B) Cliché de puissance supérieure montrant une infiltration de l'espace intervillaire par des cellules d'inflammation chronique (flèches). Les villosités chorioniques sont pour une bonne part épargnées de l'infiltration, mais montrent plutôt une vaste nécrose précoce de la couche syncytiotrophoblastique. On note aussi un dépôt de fibrine périvillaire (hématoxyline-éosine, grossissement original $\times 20$ ). (C) Cliché de forte puissance qui révèle un infarctus précoce, avec des taches dispersées et une fragmentation précoce des noyaux syncytiotrophoblastiques (flèches) (hématoxyline-éosine, grossissement original $\times 40$ ). (D) Immunocoloration des marqueurs de l'antigène CD68 pour les macrophages montrant un grand nombre de cellules d'inflammation chronique dans l'espace intervillaire, qui encerclent les villosités chorioniques (immunocoloration des marqueurs de l'antigène CD68, grossissement original ×20).

intervillite histiocytaire chronique. Ici toutefois, les signes histopathologiques étaient différents de ceux des intervillites histiocytaires chroniques typiques de 2 points de vue principalement : l'infarctus précoce étendu et les amas de cellules inflammatoires entourant les villosités chorioniques (figure 1).

La femme et le nouveau-né ont été transférés dans une chambre à pression négative de l'unité postnatale. La femme a tenté l'allaitement avec les précautions appropriées, y compris le port d'un masque, les mesures d'hygiène appropriées des mains et des seins, et en gardant le bébé à 2 mètres de distance entre les tétées. Le nouveau-né s'est révélé neutropénique et légèrement hypothermique (température la plus basse $35,9^{\circ} \mathrm{C}$ ); il a éprouvé des difficultés à s'alimenter et présenté des épisodes intermittents d'hypoglycémie (glycémie la plus basse $1,8 \mathrm{mmol} / \mathrm{L}$ ), qui ont été gérés par l'administration de gel de dextrose et de suppléments de préparations pour nourrissons. Ses épisodes répétés d'hypoglycémie et ses difficultés à s'alimenter ont nécessité son admission à l'unité des soins intensifs néonataux (USIN) à 37 heures de vie pour administration intraveineuse de glucose, thermorégulation et soutien à l'alimentation orale. Un traitement par ampicilline et tobramycine a été débuté de manière empirique (lignes directrices de routine pour la septicémie néonatale dans notre établissement); il a été cessé 48 heures plus tard après l'obtention des résultats d'hémoculture négatifs. Dans les 24 heures suivant l'admission à l'USIN, le traitement par glucose intraveineux a été cessé, le nouveau-né a maintenu sa température dans un petit lit ouvert et il a réussi à mieux s'alimenter. Aucune épreuve radiologique n'a été jugée nécessaire. Il a été ramené à la chambre de sa mère à l'unité postnatale, et la mère et l'enfant ont reçu leur congé 4 jours après la naissance.

Le nouveau-né est revenu pour un examen de routine au jour 7 . L'examen clinique s'est révélé sans particularités; le bébé ne présentait pas de difficulté à s'alimenter ni fièvre ni toux. On a répété les tests de SRAS-CoV-2 chez la mère et le nouveau-né (résultats au tableau supplémentaire S1 de l'annexe 1,). Un suivi téléphonique au jour 30 suivant la naissance a confirmé que le bébé se développait normalement. Au moment d'écrire ces lignes, un suivi continu est en place avec un médecin communautaire. 


\section{Discussion}

Des cas d'infection périnatale présumée au SRAS-CoV-2 ont été signalés ${ }^{2,3}$. Toutefois, compte tenu de la faible fréquence des tests, de la possibilité d'une contamination des spécimens à partir de foyers maternels infectés, de la validité douteuse des analyses sérologiques ${ }^{4}$ et de l'absence de définitions standardisées de la transmission congénitale, périnatale et postnatale, il faudra recueillir plus de données pour confirmer la voie de transmission ${ }^{5}$ et déterminer si une transmission in utero s'est effectivement produite.

Notre cas représente une infection congénitale probable au SRAS-CoV-2 chez un nouveau-né. Le caractère congénital de l'infection découle des observations suivantes : le nouveau-né n'a pas été en contact avec des sécrétions vaginales; les membranes étaient intactes avant la naissance; il n'y a pas eu de contacts avec la peau de la mère avant le premier prélèvement nasopharyngé chez le nouveau-né. Le phénotype clinique d'une infection au SRAS-CoV-2 chez un nouveau-né presque à terme est inconnu. Les caractéristiques cliniques de ce bébé étaient compatibles avec l'évolution d'un nouveau-né presque à terme, à l'exception de l'élévation des taux d'enzymes hépatiques. Nous avons déterminé qu'il s'agissait d'un cas probable, plutôt que confirmé, d'infection congénitale au SRAS-CoV-2 parce que les gènes cibles du SRASCoV-2 n'ont pas été détectés dans les tissus du cordon ombilical et qu'on ne disposait pas de spécimen de sang de cordon pour y faire un dépistage du SRAS-CoV-2.

Dong ${ }^{7}$ et Zeng ${ }^{4}$ et leurs collaborateurs respectifs ont identifié la présence d'anticorps anti-IgM et anti-IgG chez des nouveau-nés de femmes porteuses de la maladie à coronavirus 2019 (COVID19), tandis que, dans un autre rapport, Zeng et ses collaborateurs ${ }^{2}$ ont identifié l'ARN du SRAS-CoV-2 dans des échantillons nasopharyngés de 3 cas sur 33. La plupart des investigateurs à ce jour n'ont pas signalé la présence de l'ARN du SRAS-CoV-2 dans les sécrétions vaginales, le liquide amniotique ou le lait maternel ${ }^{8}$, sauf un cas récent où l'ARN du SRAS-CoV-2 était présent dans le liquide amniotique ${ }^{9}$. Ici, le prélèvement nasopharyngé chez la mère, de même que le lait maternel et les sécrétions vaginales étaient positifs à l'égard de l'ARN du SRAS-CoV-2. La contamination potentielle du lait maternel par les sécrétions respiratoires ne peut pas être écartée, mais le risque a été minimisé par une bonne hygiène des seins avant le prélèvement. Le résultat positif pour le prélèvement vaginal pourrait résulter d'une inoculation par les selles, mais dans le contexte des résultats positifs pour le placenta, le parenchyme placentaire et le tissu chorionique, il est plus probablement question ici d'excrétion virale à partir du lit placentaire présumément infecté. Le SRAS-CoV-2 infecte les cellules hôtes par le biais des protéines membranaires de l'enzyme de conversion de l'angiotensine $2^{10}$, qui est fortement exprimée dans le placenta11,12. L'homogénéité temporelle des observations et la présence d'un infarctus précoce évoquent une atteinte diffuse de tout le placenta, qui concorde davantage avec une infection virale primaire qu'avec une surinfection du placenta sur fond d'intervillite histiocytaire chronique. Étant donné que les gènes du SRAS-CoV-2 n'ont pas été détectés dans le tissu ombilical, nous soupçonnons la possibilité d'une voie de transmission transamniotique par le placenta.
Le diagnostic d'une infection congénitale repose habituellement sur des caractéristiques cliniques compatibles et sur la présence d'un agent pathogène ou d'un anticorps dans le lait maternel, le sang de cordon, le liquide amniotique, le placenta et chez le nouveau-né. Le débat quant à la transmission par le sang ou durant l'accouchement a toujours cours ${ }^{5}$. Des tests complets effectués sur-le-champ sont nécessaires pour comprendre la voie de transmission du SRAS-CoV-2.

Dans ce cas-ci, la neutropénie familiale chez la mère et l'immunodépression associée peuvent avoir contribué à la dissémination du virus dans tous les tissus et toutes les sécrétions de l'organisme. Il est possible que le nouveau-né ait hérité de la neutropénie maternelle (les résultats des tests sont attendus), ce qui peut avoir modifié son statut immunologique et contribué à l'acquisition de l'infection au SRAS-CoV-2.

L'infection congénitale au SRAS-CoV-2, avec présence du virus dans le nasopharynx du nouveau-né à la naissance, peut survenir, mais la fréquence du phénomène est inconnue. Tous les professionnels de la santé présents lors de l'accouchement d'une femme infectée et assignés aux soins au nouveau-né à l'USIN devraient être au courant de ce risque et utiliser l'équipement de protection individuelle approprié pour prévenir la transmission par aérosols, gouttelettes et contact, dès que des interventions générant des aérosols sont envisagées. Chez les nouveau-nés, il faut procéder le plus rapidement possible au dépistage de l'ARN du SRAS-CoV-2 dans le sang de cordon, les spécimens placentaires et le nasopharynx, sans attendre les 24 heures préconisées dans les lignes directrices actuelles de l'American Academy of Pediatrics ${ }^{13}$. Cela permettrait d'établir la prévalence du SRAS-CoV-2 chez les nouveau-nés de mères infectées et la classification des cas en fonction de l'étape du processus (in utero, périnatal ou postnatal) et non de la direction (verticale ou horizontale) de la transmission ${ }^{6}$. Cela est important également pour la prise en charge du nouveauné et la surveillance des effets différés de la transmission chez le nourrisson. En terminant, il est important de consigner le moment, le processus et la technique et des premiers prélèvements d'échantillons afin d'identifier toute transmission périnatale (par opposition à une infection congénitale vraie [in utero]) et d'appliquer les précautions appropriées pour protéger le personnel soignant. L'obtention rapide des résultats peut aussi aider à gérer les ressources nécessaires aux soins de la mère et de l'enfant. L'obtention de 2 tests de SRAS-CoV-2 consécutifs négatifs pour le nouveau-né permettra au personnel soignant de cesser les précautions contre la transmission par gouttelettes et contact; toutefois, dans certaines unités, le bébé continuera d'être considéré comme un contact potentiellement contagieux pendant 14 jours.

\section{Références}

1. Vlachodimitropoulou Koumoutsea E, Vivanti AJ, Shehata N, et al. COVID-19 and acute coagulopathy in pregnancy. J Thromb Haemost le 17 avril 2020. [Cyberpublication avant impression]. doi: 10.1111/jth.14856.

2. Zeng L, Xia S, Yuan W, et al. Neonatal early-onset infection with SARS-CoV-2 in 33 neonates born to mothers with COVID-19 in Wuhan, China. JAMA Pediatr le 26 mars 2020. [Cyberpublication avant impression]. doi: 10.1001/jamapediatrics.2020.0878.

3. Izamora MC, Paredes T, Caceres D, et al. Severe COVID-19 during pregnancy and possible vertical transmission. Am J Perinatol le 18 avril 2020. [Cyberpublication avant impression]. doi: 10.1055/s-0040-1710050. 
4. Zeng $\mathrm{H}$, Xu C, Fan J, et al. Antibodies in infants born to mothers with COVID-19 pneumonia. JAMA le 26 mars 2020. [Cyberpublication avant impression]. doi: 10.1001/jama.2020.4861.

5. Kimberlin DW, Stagno S. Can SARS-CoV-2 infection be acquired in utero?: More definitive evidence is needed. JAMA le 26 mars 2020. [Cyberpublication avant impression]. doi: 10.1001/jama.2020.4868.

6. Shah PS, Diambomba Y, Acharya G, et al. Classification system and case definition for SARS-CoV-2 infection in pregnant women, fetuses, and neonates. Acta Obstet Gynecol Scand le 11 avril 2020. [Cyberpublication avant impression]. doi: 10.1111 /aogs.13870

7. Dong L, Tian J, He S, et al. Possible vertical transmission of SARS-CoV-2 from an infected mother to her newborn. JAMA le 26 mars 2020. [Cyberpublication avant impression]. doi: 10.1001/jama.2020.4621.

8. Chen H, Guo J, Wang C, et al. Clinical characteristics and intrauterine vertical transmission potential of COVID-19 infection in nine pregnant women: a retrospective review of medical records. Lancet 2020;395:809-15.
9. Zamaniyan M, Ebadi A, Aghajanpoor Mir S, et al. Preterm delivery in pregnant woman with critical COVID-19 pneumonia and vertical transmission. Prenat Diagn le 17 avril 2020. [Cyberpublication avant impression]. doi: 10.1002/pd.5713.

10. Xu H, Zhong L, Deng J, et al. High expression of ACE2 receptor of 2019-nCoV on the epithelial cells of oral mucosa. Int J Oral Sci 2020;12:8.

11. Levy A, Yagil Y, Bursztyn M, et al. ACE2 expression and activity are enhanced during pregnancy. Am J Physiol Regul Integr Comp Physiol 2008;295:R1953-61.

12. Li M, Chen L, Zhang J, et al. The SARS-CoV-2 receptor ACE2 expression of maternal-fetal interface and fetal organs by single-cell transcriptome study. PLoS One 2020;15:e0230295.

13. Puopolo KM, Hudak ML, Kimberlin DW, et al. Initial guidance: management of infants born to mothers with COVID-19. Washington: American Academy of Pediatrics Committee on Fetus and Newborn, Section on Neonatal Perinatal Medicine and Committee on Infectious Diseases; le 2 avril 2020. Accessible ici : https://downloads.aap.org/AAP/PDF/COVID\%2019\%20Initial\%20Newborn\%20 Guidance.pdf (consulté le 15 avril 2020).
Intérêts concurrents : Susan Poutanen déclare avoir reçu des honoraires de Merck en lien avec des comités consultatifs et des conférences, des honoraires de Verity, Cipher Pharmaceuticals et Paladin Labs en lien avec des comités consultatifs, un remboursement partiel de frais de déplacement lors de conférences de COPAN Diagnostics et un soutien à la recherche de Accelerate Diagnostics et bioMérieux, tous sans lien avec le présent article. Ann Malinowski est membre du comité consultatif d'Alexion sur la microangiopathie thrombotique postnatale et a participé à une conférence commandée par Alexion sur la microangiopathie thrombotique dans la grossesse. Aucun autre intérêt concurrent n'a été déclaré.

Cet article a été révisé par des pairs.

Les auteurs ont obtenu le consentement de la patiente.

Affiliations : Services de pédiatrie (Kirtsman, Diambomba, Shah), d'obstétrique et gynécologie Malinowski, Vlachodimitropoulou) et de pathologie et médecine de laboratoire (Parks), Hôpital du Mont-Sinaï; Services de microbiologie (Poutanen) et de médecine (Infectiologie) (Poutanen), Réseau universitaire de santé (Hôpital du Mont-Sinaï); Départements de pédiatrie (Kirtsman, Diambomba, Erdman, Morris,
Shah), de médecine de laboratoire et pathobiologie (Poutanen, Parks), de médecine (Poutanen) et d'obstétrique et gynécologie (Malinowski, Vlachodimitropoulou), Université de Toronto; Division d'infectiologie (Erdman, Morris), Service de pédiatrie, Hôpital pour enfants malades de Toronto, Toronto, Ont.

Collaborateurs : Maksim Kirtsman et Prakesh Shah ont rédigé le manuscrit. Tous les auteurs ont contribué également à la conception et à l'élaboration du travail et révisé de façon critique le contenu intellectuel important; ils ont donné leur approbation finale pour la version destinée à être publiée et assument l'entière responsabilité de tous les aspects du travail.

Remerciements : Les auteurs remercient Heather McDonald Kinkaid, Centre de recherche mère-enfant, Hôpital du Mont-Sinaï, Toronto, pour son aide éditoriale lors de la préparation du manuscrit. Ils remercient également la famille qui a donné son consentement à la publication de ce cas.

Correspondance : Prakesh Shah, prakeshkumar.shah@ sinaihealthsystem.ca 